\title{
Irreversible Alteration of Extracellular Vesicle and Cell-Free Messenger RNA Profiles in Human Plasma Associated With Ex Vivo Platelet Activation
}

Hyun Ji Kim

Oregon Health \& Science University

Matthew Rames

Oregon Health \& Science University

Samuel Tassi Yunga

Oregon Health \& Science University

Randall Armstrong

Oregon Health \& Science University

Mayu Morita

Oregon Health \& Science University

Anh Ngo

Oregon Health \& Science University

Owen McCarty

Oregon Health \& Science University

Fehmi Civitci

Oregon Health \& Science University

Terry Morgan

Oregon Health \& Science University

Thuy Ngo ( $\square$ ngth@ohsu.edu )

Oregon Health \& Science University

\section{Research Article}

Keywords: RT-qPCR, mRNA (cf-mRNA), clinically relevant circulating biomarkers

Posted Date: January 4th, 2021

DOI: https://doi.org/10.21203/rs.3.rs-133629/v1

License: (1) (1) This work is licensed under a Creative Commons Attribution 4.0 International License.

Read Full License 


\section{Abstract}

The discovery and utility of clinically relevant circulating biomarkers depend on standardized methods that minimize preanalytical errors. Here, we examined the preanalytical variations of extracellular vesicles (EVs) and cell-free mRNA (cf-mRNA) profiles in human plasma and how post-freeze/thaw processing removes and retains EVs and cf-mRNA subtypes. Using multiparametric nanoscale flow cytometric analysis, which utilized forward light scatter, side scatter, and fluorescence intensity, we characterized the effect of specific preanalytical variables on vesicle subpopulations in human plasma. Cf-mRNA levels were measured by multiplexed RT-qPCR with a panel including housekeeping, platelet, and tissue-specific genes. We found that blood processing centrifugation and temperature strongly impacted the levels of residual platelets and platelet microvesicles. Intriguingly, exosome-sized events were more resistant to processing methods. We also examined the morphology of quiescent and activated platelets and plateletderived EVs by electron microscopy. We demonstrated that platelet activation and fragmentation generated ex vivo vesicles similar to EVs in size, morphology, and canonical surface markers. Critically, freezing plasma containing residual platelets yielded irreversible ex vivo generation of new EV subpopulations and cf-mRNA transcripts, which were not removable by additional processing after freeze-thaw. In summary, we identified distinct subpopulations of EVs and cf-mRNA in human plasma that are differentially influenced by platelet activation and fragmentation during sample collection, processing, and banking via post freeze/thaw processing.

\section{Introduction}

Circulating extracellular vesicles (EVs) and cell-free RNA (cfRNA) are promising biomarkers for early cancer diagnosis [1]. EVs are a heterogeneous mixture of vesicles of varying size and composition that are released from cells either through the endosomal multivesicular body pathway, or by exophytic budding from the plasma membrane [2-5]. Based on their relative size and biogenesis, EVs are subdivided into exosome- and microvesicles. Exosomes represent a specific subtype of EVs, between $30-150 \mathrm{~nm}$ in size which originate from the inward budding of multivesicular bodies that later fuse with the plasma membrane [6]. Microvesicles are between $0.2-1 \mu \mathrm{m}$ in diameter and are shed directly from plasma membranes [5, 7]. Since EVs are either derived from the plasma membrane or the involvement of multivesicular endosome fusion with the cell surface, they have cell-specific antigens on their surface that may be antibody labelled for imaging and/or isolation in -omics analyses [8-10]. Indeed, there is increasing evidence that EVs may transport a variety of proteins and nucleic acids, including being a potential carrier of cfRNA $[11,12]$. Cell-free messenger RNAs (cf-mRNA) specifically are protein coding mRNA molecules in plasma that may serve as biomarkers $[13,14]$. Since EVs may transport diverse extracellular RNAs, including cf-mRNA, there is an intense interest in the combination of these analytes for blood-based cancer diagnosis $[15,16]$.

Previous studies have suggested that ex vivo platelet activation and fragmentation affect EV profiles in serum and plasma [2,17-19, 21-24]. Canonical exosomal-related antigens like tetraspanin proteins CD9 and CD63 are also present on platelets and platelet-derived EVs, complicating the discernment of in vivo 
versus ex vivo derived EVs in plasma [25-27]. The International Society of Extracellular Vesicles (ISEV) and International society on Thrombosis and Haemostasis (ISTH) have recommended general plateletpoor plasma processing conditions for EV analysis [28, 29]. However, how specific preanalytical variables can influence EV subpopulations is not thoroughly understood. Others have shown that residual platelets also significantly affect plasma microRNA levels solely due to differences in blood processing methods $[18,19]$. However, no prior studies have specifically examined changes in cf-mRNA. Since common blood processing conditions for biobanking may not produce platelet-poor plasma as guided by ISEV and ISTH, additional processing on banked samples after thawing may mitigate the effect of platelet activation on EVs and cf-mRNA analysis. However, which subpopulations of ex vivo generated EVs and cf-mRNA subtypes are removable or retained is unknown.

Flow cytometry has been utilized to more precisely characterize the heterogeneity of EV subpopulations by relative sizes and surface markers, as this method can simultaneously image and count EVs of different size ranges from a variety of cell types [2, 18, 30-35]. Nonetheless, standardizing nanoscale flow cytometry for sub-micron sized EV detection can be challenging due to varying instrument settings and resolution [32-34]. In addition, similar surface proteins may confound the identification of different subpopulations of EVs and fragmented residual platelets [30,36]. Therefore, distinguishing in vivo platelet-derived EVs from ex vivo platelet artifacts is critical to understand the impact of blood processing on EV and -omics analyses.

In this study, we systematically examined the variation of both EV and cf-mRNA subpopulations in human plasma due to blood processing preanalytical influences. As a proof of principle to delineate the impact of residual platelets specifically, we compared plasma derived from single spin $(1,000 \times \mathrm{g}$ centrifugation) and double spin (15,000 $\times \mathrm{g}$ secondary spin after the initial single spin). To investigate the influence of ex vivo platelet activation, we compared room temperature to cold preparations and the freeze-thawing of aliquots prepared using varying centrifugation speeds. We examined how post freeze/thaw processing removes and retains EV subpopulations as well as cf-mRNA originated from platelets, common cell types and tissue specific cells. By applying hierarchical clustering to cf-mRNA profiles, we found three cf-mRNA groups that were differentially affected by preanalytical variables. The clusters included 1) tissue-specific genes independent of blood processing conditions and genes common to many organs and cell types which are either 2) completely or 3) partially removed. Our analysis revealed EV subpopulations and cf-mRNA levels were irreversibly altered ex vivo as a consequence of the presence, activation and fragmentation of residual platelets.

\section{Materials And Methods}

\section{Blood sample collection and processing}

All experimental protocols were reviewed and approved by the Oregon Health \& Science University Institutional Review Board. All methods were carried out in accordance with relevant guidelines and regulations. Blood samples from healthy individuals were obtained from the Cancer Early Detection 
Advanced Research center (CEDAR) at Oregon Health and Science University. All samples were collected under institutional review board (IRB) approved protocols with informed consent from all participants for research use. Whole blood was collected from healthy individuals in $10 \mathrm{ml}$ in K2EDTA tubes (BD Vacutainer, Becton Dickinson, catalog number: 36643 ), $4.5 \mathrm{ml}$ in 3.2\% Sodium Citrate (NaCit) tubes (BD Vacutainer, Becton Dickinson, catalog number: 369714), and $10 \mathrm{ml}$ in sodium heparin tubes (BD Vacutainer, Becton Dickinson, catalog number: 367874 ) via antecubital vein puncture using a 21G butterfly needle (BD Vacutainer, Becton Dickinson, catalog number: 367281). For NaCit/ACD tubes, we first made acid-citrate-dextrose (ACD) by adding $12.5 \mathrm{~g}$ of sodium citrate (Sigma-Aldrich, catalog number: S4641), $10 \mathrm{~g}$ of D-glucose (Sigma-Aldrich, catalog number: G7528), and $7.5 \mathrm{~g}$ of citric acid (SigmaAldrich, catalog number: C0706) in $500 \mathrm{ml}$ of distilled water. $500 \mathrm{ul}$ of ACD solution was injected into each commercial 3.2\% NaCit tube before the blood draw. Tubes were transported vertically at room temperature before processing. Within 1 hour of blood withdrawal, plasma was prepared by centrifugation (Eppendorf 5810-R centrifuge, S-4-104 Rotor, Eppendorf) at two different processing conditions. The highest acceleration and deceleration setting was used, setting ' 9 '. $10 \mathrm{ml}$ of whole blood was spun at $1,000 \times \mathrm{g}$ for 10 minutes at $23^{\circ} \mathrm{C}(\mathrm{S} 1)$. Supernatant was collected until $10 \mathrm{~mm}$ above the buffy coat. The second centrifugation was done at $15,000 \times \mathrm{g}$ for 10 minutes at $23^{\circ} \mathrm{C}$ in $1.5 \mathrm{ml}$ microcentrifuge tubes (VWR, catalog number: 89126-714). Resulting supernatants of platelet-depleted plasma were aliquoted to new $1.5 \mathrm{ml}$ microcentrifuge tubes (VWR, catalog number: 89126-714) and immediately stored at $-80^{\circ} \mathrm{C}$. Age and gender status were recorded for all individuals. Unless indicated, plasma was processed at RT in EDTA.

\section{Sample preparation}

\section{(i) Platelet isolation from peripheral blood}

For platelet marker studies, $10 \mathrm{ml}$ of citrated whole blood was centrifuged at $200 \times \mathrm{g}$ for $20 \mathrm{~min}$ at room temperature without any brakes. $10 \mu \mathrm{l}$ of $10 \mathrm{mg} / \mathrm{ml}$ prostaglandin $\mathrm{I}_{2}\left(\mathrm{PGI}_{2}\right)$ was added to the upper two thirds of platelet-rich plasma (PRP). PRP was centrifuged at $1,000 \times \mathrm{g}$ for $10 \mathrm{~min}$ at room temperature to pellet the platelets. $1 \mathrm{ml}$ of Tyrode's buffer (TB) was added with $150 \mathrm{ml}$ of acid citrate dextrose (ACD) to resuspend the platelets. To wash the platelets, extra TB was added up to a total of $25 \mathrm{ml}$ followed by $3 \mathrm{ml}$ of $A C D$ and $10 \mu \mathrm{l}$ of $\mathrm{PGI}_{2}$. The platelet suspension was centrifuged at $1,000 \times \mathrm{g}$ for $10 \mathrm{~min}$ and resuspended in $1 \mathrm{ml}$ of TB. The washed platelets were counted using a haemocytometer and resuspended to final concentration of $10^{9}$ platelets $/ \mathrm{ml}$.

\section{(ii) Platelet activation to induce platelet-derived EV formation}

For platelet derived EV studies, whole blood collected in EDTA was centrifuged at $200 \times \mathrm{g}$ for $20 \mathrm{~min}$ without any brakes to collect platelet-rich plasma (PRP). PRP was collected and centrifuged again at $1,000 \times \mathrm{g}$ for $10 \mathrm{~min}$ at RT to pellet resting platelets. The platelet pellet was resuspended in Tyrode's buffer (TB) pre-warmed to $37^{\circ} \mathrm{C}$ and centrifuged at $200 \times \mathrm{g}$ for $1 \mathrm{~min}$ to remove any residual red blood cells or platelet aggregates. Platelet suspension was incubated at RT, frozen at $-80^{\circ} \mathrm{C}$, or treated with 13 
$\mathrm{mM}$ ionomycin in $8 \mathrm{mM} \mathrm{CaCl}_{2}$ for $10 \mathrm{~min}$. After treatment, the platelet suspension was centrifuged at $1,000 \times \mathrm{g}$ for $10 \mathrm{~min}$ to pellet any residual platelets, and the supernatant was collected for platelet-derived EV studies. For TEM studies, the platelet suspension was recentrifuged at $1,000 \times \mathrm{g}$ for $10 \mathrm{~min}$. Isolated platelets were kept at either RT or $-80^{\circ} \mathrm{C}$ for $10 \mathrm{~min}$ and resuspended before imaging.

\section{Platelet counting}

For counting platelets in differentially processed plasma, blood samples from three healthy individuals were obtained in $10 \mathrm{ml}$ K2EDTA tubes (BD Vacutainer, Becton Dickinson, catalog number: 36643). Plasma was processed using single spin at $1,000 \times \mathrm{g}$ (S1) and double spin at 15,000 $\mathrm{xg}$ (S2) both at either room temperature or at $4^{\circ} \mathrm{C}$ to compare with the standard platelet-rich plasma (PRP) processing protocol at $200 \mathrm{x} \mathrm{g}$ for $20 \mathrm{~min}$ at room temperature. The platelet count was measured by the improved Neubauer haemocytometer (VWR Scientific Products, Piscataway, NJ) by two independent, experienced researchers. The total number of platelets were counted from central $1 \times 1 \mathrm{~mm}$ area consisting of 25 groups of 16 squares separated by closely ruled triple lines, equivalent to a volume of $0.1 \mu \mathrm{l}$.

\section{Fluorescent antibody labeling of whole blood, isolated platelets, and EVs for flow cytometry}

To fluorescently label myeloid cells, leukocytes, and platelets, $100 \mathrm{ml}$ of whole blood was stained with 2 $\mathrm{ml}$ of each antibody: Brilliant Violet-650 conjugated anti-CD45 (clone HI30, Biolegend Catalog number 304042), FITC-conjugated anti-CD41a (Biolegend, catalog number 303730), Phycoerythrin (PE)conjugated anti-CD9 (Biolegend, catalog number 312106), AlexaFluor 488-conjugated anti-CD63 (Thermo Fisher Scientific, clone MEM-259, catalog number MA5-18149), and PE-conjugated anti-CD81 (Thermo Fisher Scientific, clone M38, catalog number A15781). Prior to flow cytometric analysis, red blood cells (RBCs) were lysed by the addition of $1 \mathrm{ml}$ of 1 X RBC lysis buffer (Invitrogen, catalog number 00433357). To fluorescently label purified platelets, $40 \mathrm{ml}$ of resuspended isolated platelets were stained with $2 \mathrm{ml}$ of each respective antibody and incubated with $50 \mathrm{ml}$ of TB for $15 \mathrm{~min}$ at room temperature on a $200 \mathrm{rpm}$ shaker. For thrombin activation, bovine thrombin was added to a final concentration of $0.1 \mathrm{U} / \mathrm{ml}$. After incubation, platelets were fixed by adding $100 \mathrm{ml}$ of $2 \%$ Paraformaldehyde (PFA) for 10 minutes. Fixed platelets were diluted with $300 \mathrm{ml}$ of D-PBS prior to flow cytometric analysis.

To fluorescently label EV surface proteins, $5 \mu$ l of plasma, isolated extracellular vesicles and buffer were incubated with $5 \mu$ l of antibody mix prepared after established dilution series. CD41a BV421 (Biolegend, clone: HIP8, catalog number: 303730 , concentration: $0.1 \mathrm{mg} / \mathrm{ml}$ ) was diluted to a final concentration of $0.0005 \mathrm{mg} / \mathrm{ml}-0.001 \mathrm{mg} / \mathrm{ml}$ for staining. CD81 PE (Thermofisher scientific, clone: M38, catalog number: A15781, concentration: $0.3 \mathrm{mg} / \mathrm{ml}$ ) was diluted to a final concentration of $0.012 \mathrm{mg} / \mathrm{ml}$ for staining. CD63 alexa fluorophore 488 (Thermofisher scientific, clone: MEM-259, catalog number: MA5-18149, concentration $0.26 \mathrm{mg} / \mathrm{ml}$ ) was diluted to a final concentration of $0.0026 \mathrm{mg} / \mathrm{ml}$ for staining. CD9 alexa

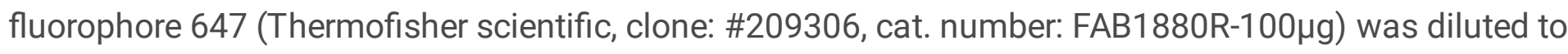
a final concentration of 0.001 to $0.002 \mathrm{mg} / \mathrm{ml}$ for staining. Incubation was done for 3 hours at room temperature in the dark. Prior to flow cytometry, the stained EV samples were further diluted with $0.1 \mathrm{~mm}$ 
filtered D-PBS without calcium and magnesium (Thermo Fisher Scientific, catalog number: 14190250) to limit the "swarming" effect using an abort rate of $<5 \%$ and keeping the threshold rate below 20,000 events per second. A buffer-only control of $0.1 \mu \mathrm{m}$-filtered DPBS without calcium and magnesium (Thermo Fisher Scientific, catalog number: 14190250) was recorded at the same flow cytometer acquisition settings as all other samples, including triggering threshold, voltages, and flow rate. The buffer-only control had a count of $<500$ events per second.

\section{Flow cytometry set-up for platelets, microvesicles, and exosomes}

For whole blood and isolated platelet marker studies, a Becton-Dickinson FACSymphony equipped with $200 \mathrm{~mW} 488 \mathrm{~nm}, 561 \mathrm{~nm}, 405 \mathrm{~nm}$ and $640 \mathrm{~nm}$ lasers was used. For each sample, the SSC trigger threshold value was set to 200 . The sample flow rate was set at low, approximately $12 \mathrm{ml} / \mathrm{minute}$, and the data was collected for 60 seconds. For submicron size detection of smaller platelets, microvesicles, and exosome-sized nanoparticles, Beckton-Dickinson FACSAria Fusion equipped with $488 \mathrm{~nm}(60 \mathrm{~mW})$, $561 \mathrm{~nm}(100 \mathrm{~mW}), 405 \mathrm{~nm}(100 \mathrm{~mW})$ and 640nm (100 mW) lasers was used. For optimal configuration of submicron size detection, $0.1 \mu \mathrm{m}$ size filter was applied to the sheath fluidic system to reduce sheath fluid noise. Data collection was set using the SSC trigger threshold value of 200 and analyzed using a log scale SSC-A to adjust for the geometric increase in light scatter related to particle diameter and refractive index. Voltage was set based on aligning $200 \mathrm{~nm}$ Megamix beads at SSC intensity of $10^{4}$. All the fluorescent voltages were set by aligning fluorescent intensity from buffer alone control to be below $2 \mathrm{x}$ $10^{2}$ for each channel. The sample flow rate was set at 1 , which is approximately $10 \mathrm{ml} /$ minute. Timed collections were recorded for 60 seconds in repeated experiments with high reproducibility within and between BD flow cytometers (FACSAria and FACSymphony). To account for the electronic abort rate due to nanoparticle coincidence within nano-droplets being imaged (also known as "swarming"), stained samples were diluted to retain the threshold event rates below 20,000 events per second with less than a $5 \%$ abort rate and validated via consistent median fluorescent intensity across plasma dilutions (Supplementary Fig. 3A). Megamix-plus FSC and SSC (BioCytex, catalog number 7802 and 7803) polystyrene calibration beads $(100,160,200,240,300,500$, and $900 \mathrm{~nm})$ conjugated to green fluorescence were measured using the $488 \mathrm{~nm}$ laser. All experiments were standardized to the $200 \mathrm{~nm}$ bead SSC-A and $900 \mathrm{~nm}$ bead $488 \mathrm{~nm}$ fluorescence (similar to using MESF beads). SSC signal intensity for the $200 \mathrm{~nm}$ beads was set at $10^{4}$ for every experiment. This ensured comparable fluorescence and light scatter measurements between experiments. We analyzed plasma stained with CD41a, CD63, CD81, and CD9 markers using FSC/SSC/FL dot plots. All measurements were analyzed using FlowJo software in bi-exponential scale for forward, side-scatter, and fluorescence intensities. Events whose SSC intensity was less than $10^{4}$ were labelled as exosome-sized (EXO) populations. Events whose SSC intensity was greater than $10^{4}$ were back-gated onto SSC/FSC dot plot, further differentiating into $\mathrm{FSC}^{+}$and $\mathrm{FSC}^{-}$ subsets defined as platelet (PLT/MV) and microvesicles (MV), respectively.

\section{Transmission electron microscopy}


Ultrathin carbon film on lacey carbon support with 400 mesh on copper (Ted Pella, catalog number: 01824) was glow discharged for 30 seconds using PELCO easiGlow glow discharger (Ted Pella). Isolated platelet samples (RT vs. $-80^{\circ} \mathrm{C}$ ) were put on charged grids for $1 \mathrm{~min}$, washed for 30 seconds with MilliQ water, and fixed with $1 \%$ uranyl acetate for 30 seconds. Grids with stained samples were air dried at least 30 minutes before imaging. Prepared samples were imaged at $120 \mathrm{kV}$ using FEI Tecnai ${ }^{\mathrm{TM}}$ Spirit TEM system. FEl- Tecnai ${ }^{\text {TM }}$ Spirit TEM system was interfaced to a bottom mounted Eagle ${ }^{\text {TM }} 2 \mathrm{~K}$ TEM CCD multiscan camera and to a NanoSprint12S-B cMOS camera from Advanced Microscopy Techniques (AMT) fast side mounted TEM CCD Camera. Images were collected at 8,000-80,000x magnification under $1-2 \mu \mathrm{m}$ defocus. Regions shown in Figure 3 were representative of each sample, which were uniformly observed from larger fields of view across the grids. Images were acquired as $2048 \times 2048$ pixel, 16-bit gray scale files using the FEl's TEM Imaging \& Analysis (TIA) interface on an Eagle ${ }^{\mathrm{TM}} 2 \mathrm{~K}$ CCD multiscan camera.

\section{RT-qPCR profiling of cell free mRNA}

For characterizing the effect of freeze thaw on cell free mRNA expressions, RNA was extracted using plasma processed with S1, S2, S1FR, S2FR, and S1FRS2 conditions. Cell free mRNA was isolated by using plasma/serum circulating and exosomal RNA purification Kit (Norgen Biotek) followed by 10X Baseline-ZERO DNase treatment (Epicentre). DNase treated RNA samples were purified and further concentrated using RNA clean and concentrator (Zymo Research). The purified RNA samples were assayed by RT-qPCR using 16 primers (MTND2, PPBP, B2M, PF4, ACTB, CORO1C, GSE1, GAPDH, SMC4, HBG1, NUSAP1, MIKI67, FGB, APOE, FGG, and ALB). Template RNA was mixed with Superscript III Onestep RT-PCR system with Platinum Taq DNA polymerase (Invitrogen) to generate CDNA according to the protocol. PCR amplification products were treated with Exonuclease I (New England Biolabs) to digest single stranded primers at $37^{\circ} \mathrm{C}$ for $30 \mathrm{~min}$ followed by inactivation of enzymes at $80^{\circ} \mathrm{C}$ for $15 \mathrm{~min}$. For RT-qPCR, cDNA from preamplification was diluted 1:80 and set-up in 96-well plates with SsoFast EvaGreen supermix with low ROX (BioRad) with above primers at 10 M. QuantStudio 7 Flex (Applied Biosystems) was used to run RT-qPCR assay according to manufacturer's recommended cycling conditions.

\section{Statistical Analysis}

To determine the impact of overall preanalytical factors including spin, temperature, and types of anticoagulant tubes, statistical analysis was performed on marker specific events from the flow cytometry data. The significance of individual preanalytical factor comparisons were determined using pair-wise Wilcoxon rank sum test. To compare average platelet-derived EV counts associated with ionomycin or temperature $\left(-80^{\circ} \mathrm{C}\right)$ induced platelet activation, pair-wise Wilcoxon rank sum test was performed on marker specific events from flow cytometry analysis. To determine the effect of freeze thaw on extracellular vesicles, flow cytometric counts of exosomes per different stained surface markers $\left(\mathrm{CD} 41 \mathrm{a}^{+}, \mathrm{CD} 63^{+}\right.$, and $\left.\mathrm{CD} 41 \mathrm{a}^{+} / \mathrm{CD} 63^{+}\right)$were normalized across pre/post-thaw processing conditions. To determine the significance of freeze-thaw effects in pre/post-thaw processing conditions, Tukey's multiple 
comparison test was performed on normalized counts. Analyses were conducted using GraphPad Prism and $\mathrm{R}$ package.

\section{Results}

\section{Multiparametric nanoscale flow cytometry reveals distinct platelet and vesicle populations in human plasma}

To analyze potential residual platelet and vesicle populations in plasma derived from single spin ( $\mathrm{S} 1$, spun at 1,000 $\times \mathrm{g}$ ) and double spin (S2, second spin at 15,000 $\times \mathrm{g}$ ), we utilized nanoscale flow cytometry [37]. Light scatter (side scatter and forward scatter) and fluorescence (FL) were calibrated using commercially available polystyrene beads ranging in diameter from 100-900 nm [33]. The submicron sized bead mixture was resolved into distinct clusters based on their sizes (Figure 1A). We standardized side scatter (SSC) intensity between experiments by setting the $200 \mathrm{~nm}$ bead at an intensity of $10^{4}$ for every experiment (Figure 1A-F, Supplementary Figure 1A). We observed that forward light scatter (FSC) could only resolve bead sizes larger than $900 \mathrm{~nm}$ on our FACSAria or FACSymphony instruments using a $488 \mathrm{~nm}$ laser (Figure 1A, Supplementary Figure 1B). To define the gates for residual platelets and EV subpopulations, we used purified quiescent platelets (Figure 1B and Supplementary Figure 1) and EVs derived from activated platelets at $-80^{\circ} \mathrm{C}$ with remnant platelets removed by subsequent centrifugation (Figure 1C and Supplementary Figure 1). Isolated platelets were identified as positive for the platelet marker (CD41a) and distinguished by a high FSC (Figure 1B). The platelet-derived EV preparation also had $\mathrm{CD} 41 \mathrm{a}^{+}$events but unlike the platelet preparation, the majority of the events were FSC negative with only a small fraction having relatively high FSC (Supplementary Figure 1B). In addition, the FSC ${ }^{+}$ populations were present in single spin S1 plasma (Figure 1D) while absent in platelet-depleted S2 plasma (Figure 1E). Therefore, we referred to the $\mathrm{FSC}^{+}$gate as the PLT/MV population, which was associated with residual platelets, and likely large microvesicles and fragments derived from these platelets (Figure 1F). The platelet and MV depleted double-spun S2 plasma contained an enrichment of EVs that were generally less than $10^{4}$ as shown by the SSC/FL plot (Figure 1E). Therefore, we operationally divided the FSC ${ }^{-}$events into two gates separated at $10^{4} \mathrm{SSC}$ (or the $200 \mathrm{~nm}$ bead marker) corresponding to our MV and EXO populations (Figure 1F). In summary, we utilized multiparametric nanoscale flow cytometry analysis to subset vesicles, platelets and their derived fragments in plasma into 3 subpopulations: (1) SSC below $10^{4}$ particles without FSC, (2) SSC above $10^{4}$ without FSC and (3) SSC above $10^{4}$ with positive FSC referred hereafter as EXO, MV and PLT/MV respectively (Figure 1F, see Methods).

\section{Blood processing centrifugation speeds and temperature impact EV profiling}

To investigate the effects of preanalytical processing conditions on the three platelet related subpopulations, we compared samples prepared at room temperature with samples prepared when the temperature was kept at $4^{\circ} \mathrm{C}$ during the $\mathrm{S} 1$ and $\mathrm{S} 2$ centrifugation steps. We also compared the effect of 
various blood collection tubes known to impact platelet activation and EVs ( $\mathrm{NaCit}+\mathrm{ACD}$, NaCit alone, EDTA, or heparin) with proper assay controls [37-39]. Flow cytometry assay controls included testing unstained plasma to pre-set uniform gates, stained PBS buffer, single stained plasma controls, and multiplexed stained plasma (Supplementary Figure 2A-G). Additionally, we performed serial antibody dilutions to eliminate false positive staining and aggregates, and serial plasma dilutions to limit nanoparticle coincident detection, also known as "swarming" [37, 39]. Based on the signal-to-noise ratio from antibody dilutions, optimal antibody concentrations for the platelet-specific marker CD41a and EVrelated tetraspanin markers (CD63, CD81, CD9) were determined and uniformly employed for all experiments (Supplementary Figure 3B, C). Plasma dilution series showed the linear detection of EVs while the median FL intensity remained constant, suggesting that EVs were detected and counted as single nanoparticles within each nano-droplet analyzed via flow cytometry (Supplementary Figure 3A).

Using the above optimized flow cytometry settings and our multiparametric analysis, we assessed preanalytical influences on PLT/MV, MV, and EXO populations based on CD41a staining. We observed that differential centrifugation and processing temperature from the same anticoagulant tube type (i.e. EDTA) greatly influenced relative abundances of these populations (Figure 2A, B). Notably, back-gating of SSC $>10^{4}$ (equivalent to bead size $>200 \mathrm{~nm}$ ) vs CD41a fluorescence (FL) onto SSC vs FSC shows the two distinct population s of PLT/MV and MV (low FSC) in S1, which are lost in the 15,000 $\times \mathrm{g}$ S2 preparations (Figure 2A, B). To further characterize the influence of individual preanalytical factors, counts from the three distinct platelet-related populations were compared (Figure $2 \mathrm{C}-\mathrm{H}$ ). The overall differences in $\mathrm{CD} 41 \mathrm{a}^{+}$ $\mathrm{PLT} / \mathrm{MV}, \mathrm{MV}$, and EXO counts were related to differential centrifugation speeds as expected (Figure 2C, F), while the blood processing temperature strongly influenced relative CD $41 \mathrm{a}^{+} \mathrm{PLT} / \mathrm{MV}$ and MV populations in the $S 1$ condition but not S2 (Figure 2D, G). We suspect this could be related to residual platelet activation making more ex vivo $\mathrm{MVs}$ at $4^{\circ} \mathrm{C}$ or increased platelet sedimentation at room temperature. To systematically evaluate residual platelet amount in each processing condition, we counted platelets in S1 and S2 plasma compared to the standard platelet-rich plasma (PRP) processing protocol using a haemocytometer. We found an average platelet concentration of $354 \pm 68$ thousand/ $\mu \mathrm{l}$ in PRP, $313 \pm 74$ thousand/ $\mu \mathrm{l}$ in S1 processed at RT, and $415 \pm 62$ thousand $/ \mu \mathrm{l}$ in S1 processed at $4^{\circ} \mathrm{C}$ (Supplementary Table 1). Notably, an increase of platelet amount was observed in $\mathrm{S} 1$ processed at $4^{\circ} \mathrm{C}$ compared to RT, which agreed with our observation via multiparametric flow cytometry for the PLT/MV population. Additional secondary spin using S2 at either RT or $4^{\circ} \mathrm{C}$ resulted in the removal of more than $99.99 \%$ of residual platelets relative to PRP or S1 plasma. Conversely, the use of different anticoagulant tubes resulted in no significant differences in single spin plasma while minimal differences were found in double spin plasma (Figure 2E, H). For S2, we observed that the levels of PLT/MV and platelet-derived MVs were lower in ACD and EDTA tubes than in NaCit and Heparin. Overall, processing temperatures and differential centrifugation conditions were the major factors affecting residual platelet levels, their activation, and EV profiles.

\section{Canonical exosomal markers are expressed on plateletsandex vivo platelet-derived EVs}


To further explore the impact of residual platelets in S1 preparations (common centrifugation speed used to bank plasma in many clinical trials); we examined whole washed platelets and their associated EVs using platelet marker (CD41a) and EV tetraspanin markers (CD63, CD81, and CD9). To characterize platelet surface membrane proteins, whole blood samples containing both platelets and leukocytes were examined with SSC, pan-leukocyte marker (CD45) and the EV tetraspanin markers (Figure 3A, B). We confirmed that platelets were phenotypically distinct from leukocytes, and expressed canonical EV markers CD63 and CD9 but not CD81 at detectable levels in our assay (Figure 3A, B). Furthermore, we confirmed canonical EV marker expression on washed platelets with and without thrombin activation (Figure 3C, D). Activated platelets had higher CD63 expression than resting platelets most likely due to increased fusion of $\mathrm{CD}^{+} 3^{+}$intra-platelet granules with the platelet surface membrane during platelet activation [40] (Figure 3D).

Considering that cold temperatures induce platelet activation and the standard storage of processed plasma samples is at $-80^{\circ} \mathrm{C}$ [41], we investigated the effect of freezing on platelet-derived $\mathrm{EV}$ formation. Transmission electron microscopy (TEM) was performed on isolated platelets before and after freezing, which revealed significant freeze-thaw artifacts and the generation of EVs compared with resting platelets at room temperature (RT) (Figure 3E, F). We observed that temperature-driven platelet activation also induced ex vivo platelet-derived EV formation in the sub-500 nm range by TEM (Figure 3G), comprised of both MV and EXO-sized vesicles (Figure $3 \mathrm{H}, \mathrm{I}$ ). In order to quantify the degree of plateletderived EV formation due to freezing, we compared the relative levels of EVs directly released from isolated platelets by i) freezing at $-80^{\circ} \mathrm{C}$, ii) adding ionomycin in the presence of $\mathrm{Ca}^{2+}$ as a positive control, and iii) RT as a baseline control. The supernatants of isolated platelets before and after temperature or chemical stimulations were examined using flow cytometry. Remarkably, the number of $\mathrm{CD} 41 \mathrm{a}^{+}, \mathrm{CD} 3^{+}$or $\mathrm{CD} 9^{+} \mathrm{EV}$ s was observed to increase for platelets stored at $-80^{\circ} \mathrm{C}$ and for platelets activated with ionomycin (Figure $3 \mathbf{J}, \mathbf{K}$ ). However, CD81+ EVs remained undetectable irrespective of activation method (Figure $3 \mathbf{J}, \mathbf{K}$ ). This data suggests that platelet activation either by temperature or chemical stimulation generated substantial ex vivo platelet-derived EVs, which shared the same surface protein markers present on platelets. Our findings indicate that the presence of residual platelets and their ex vivo activation can cause significant variations in the observed amount of EVs from human plasma.

\section{Freezing of platelet containing plasma irreversibly alters EV and cf-mRNA profiles}

To further elucidate whether these artifacts may be reversed in previously banked 1,000 $\times \mathrm{g}$ (S1) plasma samples, we analyzed EV and cf-mRNA profiles in single and double spin plasma (S1 and S2) immediately or after freezing at $-80^{\circ} \mathrm{C}$ (S1FR and S2FR), or for samples subjected to a second spin following S1FR processing (S1FRS2). After freezing, the population of CD41a+ EXO significantly increased for single spin plasma (S1FR vs. $S 1, P<0.0001$ ) while remaining similar for double spin plasma irrespective of freezing (S2FR vs. S2, ns) (Figure 4A, D). The results suggest that freezing residual platelets in the single spin condition generated ex vivo platelet-derived EVs, whereas depletion of residual platelets in the double spin condition did not affect EXO counts. To test whether performing a secondary 
spin after freezing effectively removed platelet-derived EV contamination, we compared S1FRS2 relative to S1FR and S2. Compared to S1FR, S1FRS2 substantially removed CD41 ${ }^{+}$and CD63 ${ }^{+}$PLT/MV and MV populations as detected with SSC above $10^{4}$ (Figure 4A, B). Meanwhile, significantly increased levels of CD41a ${ }^{+}$EXO populations (SSC below than $10^{4}$ ) were detected even after post-thaw processing (S1FRS2 vs $S 2, \mathrm{P}<0.05$ ) (Figure 4A, D). In contrast, CD63-expressing EXO populations were statistically unchanged independent of both spinning and post-thaw processing conditions (Figure 4B, E). Intriguingly, we observed new $\mathrm{CD} 41 \mathrm{a}^{+} / \mathrm{CD} 63^{+}$costained EXO populations only after freezing single spin plasma (S1FR vs $S 1, P<0.001$ ), but not for double spin plasma (S2FR vs S2, ns) (Figure 4C, F). Collectively, this implies that platelet-derived EXO $\left(\mathrm{CD} 41 \mathrm{a}^{+}\right.$or $\left.\mathrm{CD} 41 \mathrm{a}^{+} / \mathrm{CD} 63^{+}\right)$were artificially generated from residual platelets during freeze/thaw cycles and cannot be removed by post-thaw processing (S1FRS2 vs S2FR, $P<0.05$ \& $\mathrm{P}<0.0001$ respectively). Whether this artifact confounds exosome counts from a variety of cell types in the plasma remains to be seen, but it appears to be a significant source of platelet-related ex vivo contamination.

To investigate the impact of blood sample preparation methods on cf-RNA profiles [42-44], we measured cf-mRNA transcript levels in plasma using multiplexed RT-qPCR with a panel containing housekeeping, platelet and tissue-specific genes (Figure 4G, H). Hierarchical clustering analysis of relative gene expression between post-thaw processed samples revealed three distinct clusters (Figure 4H). Overall, these clusters were either dependent (non-tissue specific) or independent (tissue specific) of post-thaw processing conditions, wherein non-tissue specific genes segregated into two clusters. The first cluster included genes (i.e. HBG1 and SMC4), that could be removed by post thaw processing and therefore were likely related to larger, micron-sized particles eliminated during S2 centrifugation (Figure $\mathbf{4 G}, \mathbf{H}$ ). The second cluster included genes (i.e. platelet gene, PF4 and house-keeping gene, B2M), that could only be partially removed by post thaw processing and therefore were likely present in smaller EVs which remained after S2 centrifugation (Figure 4G, H). Importantly, our results revealed that tissue specific gene signatures (i.e. genes expressed in liver tissue; including APOE and FGB) were retained regardless of spinning and post-thaw processing conditions (Figure $\mathbf{4 G}, \mathrm{H}$ ), suggesting they are either present in smaller non-platelet EVs or are EV-independent altogether. The relationship of cf-mRNA transcripts with EV subpopulations requires further investigation and is the subject of future studies. Overall, as genes from different biological roles are uniquely affected by preanalytical differences, the selection of novel cfRNA biomarkers should consider the effects of preanalytical variability.

\section{Discussion}

Circulating EVs and cf-RNA are promising biomarkers for disease diagnosis and prognosis [45-48]. However, significant variability in standardizing blood processing across published methods has led to a lack of reproducibility between studies $[2,18,19,49]$. In this study, we utilized multiparametric nanoscale flow cytometry and cf-mRNA profiling to characterize preanalytical influences on EV and cf-mRNA subpopulations in plasma. We observed three distinct subpopulations through multiparametric nanoscale flow cytometry differentially impacted by plasma centrifugation speeds, temperature, and 
post-thaw processing. Interestingly, we observed novel CD41a+/CD63+ costained EXO populations irreversibly introduced via freezing single spun plasma. As a first in cf-mRNA studies, we found groups of genes significantly, partially, or unaffected by post-thaw processing in plasma.

Previous studies highlighted the preanalytical influences on microparticle generation due to platelet activations [50-52]. Considerable efforts have been directed to establish a standardized methodology for platelet microparticle counting $[29,53]$. However, the enumeration of microparticles mostly utilized flow cytometry that was validated to discriminate between $0.5 \mu \mathrm{m}$ and $0.9 \mu \mathrm{m}$ Megamix beads [52, 53]. In this study, we observed variation in not only platelet-derived EVs and fragments in the size range comparable to the scatter of 0.5 to $0,9 \mu \mathrm{m}$ Megamix beads but also in smaller subpopulations. Notably, Yuana et. al. provided a comprehensive assessment of centrifugation retention for microparticles under $1.0 \mu \mathrm{m}$ utilizing flow cytometry [52]. The effect of centrifugation was performed on pre-isolated EVs from platelets and erythrocytes, but not from initial blood processing. Their freeze-thaw cycle was performed on purified EV, leading to no significant change across different temperatures of single freeze-thaw cycle. In contrast, we examined how additional processing after freeze/thaw in frozen total plasma removes or retains EVs and cf-mRNA. Therefore, we observed irreversible ex vivo generation of novel EVs and cfmRNA subpopulations associated with residual platelets in total plasma.

Since different types of EV purification methods (ultracentrifugation, density gradients, size-filtration, etc.) affect the yield and purity of EVs $[54,55]$, we chose to fluorescently label EVs directly in plasma using platelet and EV tetraspanin-specific antibodies with proper assay controls according to recent MIFlowCytEV reporting framework [37]. One limitation to our approach was the absence of size calibration particles that precisely mimic the assumed refractive index of EVs $[56,57]$. This is because the light scatter from polystyrene calibration beads with a refractive index of 1.6 is likely different from that of EVs. Although side scatter also largely depends on the diameter of the nanoparticle, knowing the precise refractive index of the specific EVs being targeted will be important for accurate sizing in future studies. Since the refractive index of specific EV populations in different buffer conditions is currently unknown, the EV subpopulations in our study are separated by relative size estimates based on the characteristics of the polystyrene beads. Specific studies, which definitively parse EV subpopulations are needed to better define EV physical characteristics and compositions.

Our heatmap analyses identified that both differential centrifugation and processing temperature significantly influenced PLT/MV and MV populations while EXO events were minimally affected. Differential centrifugation at an enhanced speed (i.e. 15,000 $\times \mathrm{g}$ ) can explain the loss of the PLT/MV and $M V$ populations in the S2 preparations. Interestingly, the MV and PLT/MV population was significantly increased by processing blood at $4^{\circ} \mathrm{C}$ compared with RT from the same donors. This observation may be attributed to the increased levels of platelet derived $\mathrm{MVs}$ at $4^{\circ} \mathrm{C}$ and the increased sedimentation rates of platelets at RT [58]. Baek et al. also tested relevant preanalytical influences on phenotypes of EVs using protein microarray [59]. However, protein microarray detects signal intensity from overall populations which hinders the ability to understand preanalytical variables on different vesicle subpopulations. Due to the significant background intensity present in different processing conditions, the increase of relative 
intensity of the cocktail array testing for CD9, CD63, and CD81 in double spun plasma is unclear [59]. Therefore, we provided a detailed instrumentation protocol with appropriate cytofluorimetric controls for reporting EV studies.

MIFlowCyt-EVguidelines have suggested sodium citrate collection tubes for EV studies [37] while others prefer EDTA tubes for -omics studies. Regardless, standardization of the collection tube type is important for the focus of downstream analysis [60]. We processed our blood samples within 1 hour of blood draw to minimize potential spontaneous ex vivo platelet activation. Accordingly, minimal effects related to the type of anticoagulant tube were observed in our plasma samples. Specifically, slightly higher MV for sodium citrate and heparin have been shown by other authors to cause less aggregation of MVs to platelets than does EDTA $[34,60]$. Our results revealed secondary spin with $15,000 \times \mathrm{g}$ centrifugation may result in the loss of platelets and platelet-derived EV aggregates from EDTA and ACD-anticoagulated samples more than from heparin and sodium citrate tubes.

Platelet activation and freeze-thaw experiments revealed that residual platelet activation confounds plasma EV and cf-mRNA analyses. The finding that both platelets and ex vivo platelet-derived EVs shared canonical EV markers (CD9, and CD63) also hinders the discernment of in vivo EVs. Indeed, our TEM studies of isolated platelets pre- and post-freeze-thaw clearly revealed temperature induced ex vivo generation of platelet-derived EVs (both MV and EXO-sized events). Generation of ex-vivo microparticles due to freeze/thaw effect was previously reported $[18,53]$. However, our freeze thaw analysis via multiparametric nanoscale flow cytometry further revealed new co-stained exosomal subpopulations $\left(\mathrm{CD} 41 \mathrm{a}^{+} / \mathrm{CD} 63^{+}\right)$, which were absent prior to freeze thaw. These novel ex vivo generated EV subpopulations add to the body of literature on platelet-associated blood processing artefacts $[2,18]$. Critically, these artificially generated EV subpopulations could not be removed by additional centrifugation after freeze/thaw, and thereby can significantly affect downstream analyses.

How blood processing influences circulating microRNA has been previously shown [18, 19], and yet the impact on cf-mRNA is poorly understood. Cheng et al. provided preanalytical influences on miRNA expression due to differing residual platelet amount [19]. Conversely, our study investigated the impact of blood processing conditions through differential centrifugation and freezing on cf-mRNA. We revealed cfmRNA groups whose extent of preanalytical variability differed based on the degree of residual platelets in plasma. In particular, non-tissue specific genes were further classified as either partially or fully removed by freeze-thaw post processing. Intriguingly, tissue-specific cf-mRNA were less prone to blood processing conditions, revealing them as potentially more robust biomarkers, or differentially associated with smaller vesicle subpopulations retained through centrifugation.

In conclusion, our study provides a comprehensive assessment of the preanalytical effect of anticoagulant tubes, centrifugation speeds, processing temperature, and freeze/thaw on plasma EVs and cf-mRNA. Employing multiparametric nanoscale flow cytometry, our work provides insights into how preanalytical factors influence vesicle subpopulations and the ex vivo release of platelet-derived EVs through residual platelet activation. Notably, these artifacts appear to be irreversible for small 
$\mathrm{CD} 41 \mathrm{a}^{+} / \mathrm{CD} 63^{+} \mathrm{EV}$ subpopulations and mRNA transcripts of genes present in platelets. Finally, we showed that there are distinct groups of cf-mRNA transcripts which were differentially influenced by preanalytical factors and post freeze/thaw centrifugation. Our results indicate that noticeable subpopulations of smaller EVs and cf-mRNA are not removable by additional spinning after freeze/thaw. Therefore, consideration should be taken when analyzing EVs and cf-mRNA from banked plasma and designing robust EV and cf-mRNA based liquid biopsy tests.

\section{Declarations}

\section{Acknowledgements}

The authors are grateful to Nick Wang of OHSU for facilitating access to Quantstudio 7 Flex Real-Time PCR systems. We are also grateful to Jeong Yoon Lim for biostatistics advice and the Cancer Early Detection Advanced Research (CEDAR) center of the OHSU Knight Cancer Institute for assistance with obtaining blood samples. We acknowledge the assistance of multiscale microscopy core for transmission electron microscopy infrastructure and thank Claudia Lopez for providing assistance in microscopy. The authors also thank Pamela S Canaday, Brianna Garcia, and Dorian Latocha for flow cytometry training at Oregon Health and Science University. We acknowledge funding support from Cancer Early Detection Advanced Research (CEDAR) center at Oregon Health \& Science University's Knight Cancer Institute, Cancer Research UK/OHSU Project Award (C63763/A27122) and by grants from the National Institutes of Health (R01HL101972, R21HD16-037, R01GM116184 and R01HL047014). The authors declare no competing financial interests.

\section{Author Contributions}

TN conceived and supervised the project. HK, FC, SY, and TN developed the initial workflow and established the blood processing optimization on EVs, platelets and cf-mRNA. TKM and MM developed the flow cytometry assay. HK, MR developed the TEM assay and designed the TEM experiments. HK, MR, FC and TN developed the analysis strategy. HK, MR, SY, RA, FC, and TN acquired and performed data analysis. HK and TN wrote the manuscript. TKM, MR, SY, FC, RA, AN, and OM edited the manuscript. All authors have reviewed and approved the manuscript.

\section{Declaration of Interest}

The authors report no conflicts of interest.

\section{References}

1. Müller, J.B., et al., Circulating biomarkers in patients with glioblastoma. British journal of cancer, 2019.

2. Marcoux, G., et al., Revealing the diversity of extracellular vesicles using high-dimensional flow cytometry analyses. Scientific Reports, 2016. 6(1): p. 35928. 
3. Maas, S.L.N., X.O. Breakefield, and A.M. Weaver, Extracellular Vesicles: Unique Intercellular Delivery Vehicles. Trends in cell biology, 2017. 27(3): p. 172-188.

4. Willms, E., et al., Extracellular Vesicle Heterogeneity: Subpopulations, Isolation Techniques, and Diverse Functions in Cancer Progression. Frontiers in immunology, 2018. 9: p. 738-738.

5. Raposo, G. and W. Stoorvogel, Extracellular vesicles: Exosomes, microvesicles, and friends. The Journal of Cell Biology, 2013. 200(4): p. 373-383.

6. Théry, C., L. Zitvogel, and S. Amigorena, Exosomes: composition, biogenesis and function. Nature Reviews Immunology, 2002. 2(8): p. 569-579.

7. Bernard, V., J. Ling, and A. Maitra, 4 - Heterogeneity of Tumor Exosomes - Role in Precision Medicine, in Diagnostic and Therapeutic Applications of Exosomes in Cancer, M. Amiji and R. Ramesh, Editors. 2018, Academic Press. p. 59-67.

8. Kalluri, R., The biology and function of exosomes in cancer. The Journal of clinical investigation, 2016. 126(4): p. 1208-1215.

9. McAndrews, K.M. and R. Kalluri, Mechanisms associated with biogenesis of exosomes in cancer. Molecular cancer, 2019. 18(1): p. 52-52.

10. György, B., et al., Membrane vesicles, current state-of-the-art: emerging role of extracellular vesicles. Cellular and molecular life sciences : CMLS, 2011. 68(16): p. 2667-2688.

11. Murillo, O.D., et al., ExRNA atlas analysis reveals distinct extracellular RNA cargo types and their carriers present across human biofluids. Cell, 2019. 177(2): p. 463-477. e15.

12. Hinger, S.A., et al., Diverse long RNAs are differentially sorted into extracellular vesicles secreted by colorectal cancer cells. Cell reports, 2018. 25(3): p. 715-725. e4.

13. Pös, O., et al., Circulating cell-free nucleic acids: characteristics and applications. European journal of human genetics : EJHG, 2018. 26(7): p. 937-945.

14. Kishikawa, T., et al., Circulating RNAs as new biomarkers for detecting pancreatic cancer. World journal of gastroenterology, 2015. 21(28): p. 8527-8540.

15. Ngo, T.T., et al., Noninvasive blood tests for fetal development predict gestational age and preterm delivery. Science, 2018. 360(6393): p. 1133-1136.

16. Lin, J., et al., Exosomes: novel biomarkers for clinical diagnosis. The scientific world journal, 2015. 2015.

17. Witwer, K.W., et al., Standardization of sample collection, isolation and analysis methods in extracellular vesicle research. Journal of extracellular vesicles, 2013. 2(1): p. 20360.

18. Mitchell, A.J., et al., Platelets confound the measurement of extracellular miRNA in archived plasma. Scientific reports, 2016. 6: p. 32651-32651.

19. Cheng, H.H., et al., Plasma processing conditions substantially influence circulating microRNA biomarker levels. PloS one, 2013. 8(6): p. e64795-e64795.

20. Heijnen, H.F., et al., Activated Platelets Release Two Types of Membrane Vesicles: Microvesicles by Surface Shedding and Exosomes Derived From Exocytosis of Multivesicular Bodies and》-Granules. 
Blood, The Journal of the American Society of Hematology, 1999. 94(11): p. 3791-3799.

21. Menck, K., et al., Isolation and Characterization of Microvesicles from Peripheral Blood. Journal of visualized experiments : JoVE, 2017(119): p. 55057.

22. Brisson, A.R., et al., Extracellular vesicles from activated platelets: a semiquantitative cryo-electron microscopy and immuno-gold labeling study. Platelets, 2017. 28(3): p. 263-271.

23. Menck, K., et al., Characterisation of tumour-derived microvesicles in cancer patients' blood and correlation with clinical outcome. Journal of Extracellular Vesicles, 2017. 6(1): p. 1340745.

24. Aatonen, M.T., et al., Isolation and characterization of platelet-derived extracellular vesicles. Journal of extracellular vesicles, 2014. 3: p. 10.3402/jev.v3.24692.

25. Venturella, M., F.M. Carpi, and D. Zocco, Standardization of blood collection and processing for the diagnostic use of extracellular vesicles. Current Pathobiology Reports, 2019. 7(1): p. 1-8.

26. Koliha, N., et al., A novel multiplex bead-based platform highlights the diversity of extracellular vesicles. Journal of Extracellular Vesicles, 2016. 5(1): p. 29975.

27. Tao, S.-C., S.-C. Guo, and C.-Q. Zhang, Platelet-derived extracellular vesicles: an emerging therapeutic approach. International journal of biological sciences, 2017. 13(7): p. 828.

28. Théry, C., et al., Minimal information for studies of extracellular vesicles 2018 (MISEV2018): a position statement of the International Society for Extracellular Vesicles and update of the MISEV2014 guidelines. Journal of Extracellular Vesicles, 2018. 7(1): p. 1535750.

29. Lacroix, R., et al., Standardization of pre-analytical variables in plasma microparticle determination: results of the International Society on Thrombosis and Haemostasis SSC Collaborative workshop. Journal of thrombosis and haemostasis : JTH, 2013: p. 10.1111/jth.12207.

30. Hargett, L.A. and N.N. Bauer, On the origin of microparticles: From "platelet dust" to mediators of intercellular communication. Pulmonary circulation, 2013. 3(2): p. 329-340.

31. Morgan, T.K., Cell- and size-specific analysis of placental extracellular vesicles in maternal plasma and pre-eclampsia. Translational Research, 2018. 201: p. 40-48.

32. Robert, S., et al., Standardization of platelet-derived microparticle counting using calibrated beads and a Cytomics FC500 routine flow cytometer: a first step towards multicenter studies? Journal of Thrombosis and Haemostasis, 2009. 7(1): p. 190-197.

33. Poncelet, P., et al., Standardized counting of circulating platelet microparticles using currently available flow cytometers and scatter-based triggering: Forward or side scatter? Cytometry Part A, 2016. 89(2): p. 148-158.

34. Jayachandran, M., et al., Methodology for isolation, identification and characterization of microvesicles in peripheral blood. Journal of immunological methods, 2012. 375(1-2): p. $207-214$.

35. Van Der Vlist, E.J., et al., Fluorescent labeling of nano-sized vesicles released by cells and subsequent quantitative and qualitative analysis by high-resolution flow cytometry. Nature protocols, 2012. 7(7): p. 1311-1326. 
36. Italiano Jr, J.E., A.T. Mairuhu, and R. Flaumenhaft, Clinical relevance of microparticles from platelets and megakaryocytes. Current opinion in hematology, 2010. 17(6): p. 578.

37. Welsh, J.A., et al., MIFlowCyt-EV: a framework for standardized reporting of extracellular vesicle flow cytometry experiments. Journal of Extracellular Vesicles, 2020. 9(1): p. 1713526.

38. Morgan, T.K., Cell-and size-specific analysis of placental extracellular vesicles in maternal plasma and pre-eclampsia. Translational Research, 2018. 201: p. 40-48.

39. Van Deun, J., et al., EV-TRACK: transparent reporting and centralizing knowledge in extracellular vesicle research. Nature methods, 2017. 14(3): p. 228-232.

40. Clemetson KJ, C.J., Platelet Receptors. Platelets, 2013. 3rd edition: p. 169-193.

41. Getz, T.M., Physiology of cold-stored platelets. Transfusion and Apheresis Science, 2019. 58(1): p. 12-15.

42. Enderle, D., et al., Characterization of RNA from Exosomes and Other Extracellular Vesicles Isolated by a Novel Spin Column-Based Method. PLOS ONE, 2015. 10(8): p. e0136133.

43. Arroyo, J.D., et al., Argonaute2 complexes carry a population of circulating microRNAs independent of vesicles in human plasma. Proceedings of the National Academy of Sciences, 2011. 108(12): p. 5003-5008.

44. Cheng, L., et al., Exosomes provide a protective and enriched source of miRNA for biomarker profiling compared to intracellular and cell-free blood. Journal of extracellular vesicles, 2014. 3(1): p. 23743.

45. Mitchell, P.S., et al., Circulating microRNAs as stable blood-based markers for cancer detection. Proceedings of the National Academy of Sciences of the United States of America, 2008. 105(30): p. 10513-10518.

46. Souza, M.F.D., et al., Circulating mRNA signature as a marker for high-risk prostate cancer. Carcinogenesis, 2019.

47. Xue, V.W., et al., Non-invasive Potential Circulating mRNA Markers for Colorectal Adenoma Using Targeted Sequencing. Scientific Reports, 2019. 9(1): p. 12943.

48. Andaloussi, S.E., et al., Extracellular vesicles: biology and emerging therapeutic opportunities. Nature reviews Drug discovery, 2013. 12(5): p. 347-357.

49. Zaporozhchenko, I.A., et al., The potential of circulating cell-free RNA as a cancer biomarker: challenges and opportunities. Expert review of molecular diagnostics, 2018. 18(2): p. 133-145.

50. Bode, A.P., et al., Vesiculation of platelets during in vitro aging. Blood, 1991. 77(4): p. 887-95.

51. Yuana, Y., R.M. Bertina, and S. Osanto, Pre-analytical and analytical issues in the analysis of blood microparticles. Thromb Haemost, 2011. 105(3): p. 396-408.

52. Yuana, Y., et al., Handling and storage of human body fluids for analysis of extracellular vesicles. Journal of extracellular vesicles, 2015. 4: p. 29260-29260.

53. Lacroix, R., et al., Impact of pre-analytical parameters on the measurement of circulating microparticles: towards standardization of protocol. J Thromb Haemost, 2012. 10(3): p. 437-46. 
54. Baranyai, T., et al., Isolation of Exosomes from Blood Plasma: Qualitative and Quantitative Comparison of Ultracentrifugation and Size Exclusion Chromatography Methods. PLOS ONE, 2015. 10(12): p. e0145686.

55. Coumans, F.A., et al., Methodological guidelines to study extracellular vesicles. Circulation research, 2017. 120(10): p. 1632-1648.

56. Gardiner, C., et al., Measurement of refractive index by nanoparticle tracking analysis reveals heterogeneity in extracellular vesicles. Journal of extracellular vesicles, 2014. 3: p. 25361-25361.

57. de Rond, L., et al., Refractive index to evaluate staining specificity of extracellular vesicles by flow cytometry. Journal of Extracellular Vesicles, 2019. 8(1): p. 1643671.

58. Ermolinskiy, P., et al., Effect of different macromolecules on viscous and microrheologic properties of blood at various temperatures. International Symposium on Optics and Biophotonics VI: Saratov Fall Meeting 2018. Vol. 11065. 2019: SPIE.

59. Bæk, R. and M.M. Jørgensen, Multiplexed Phenotyping of Small Extracellular Vesicles Using Protein Microarray (EV Array). Methods Mol Biol, 2017. 1545: p. 117-127.

60. Bæk, R., et al., The impact of various preanalytical treatments on the phenotype of small extracellular vesicles in blood analyzed by protein microarray. Journal of Immunological Methods, 2016. 438: p. 11-20.

\section{Figures}

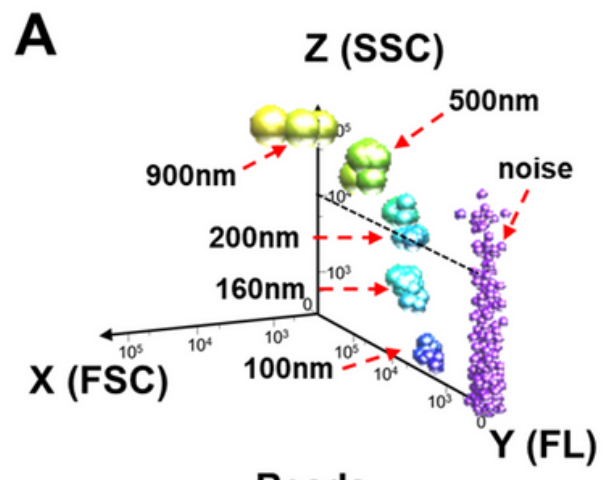

Beads

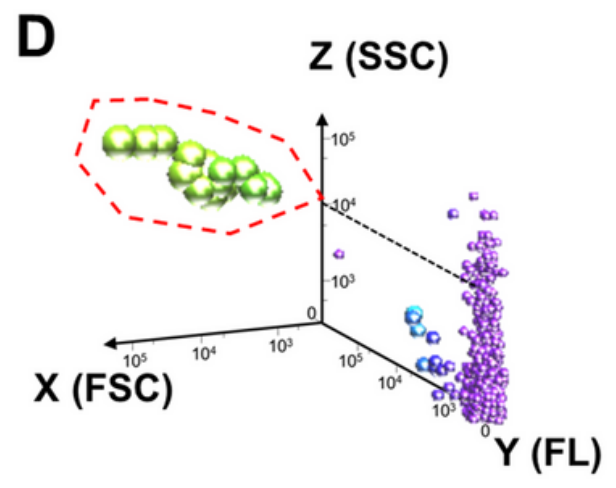

S1

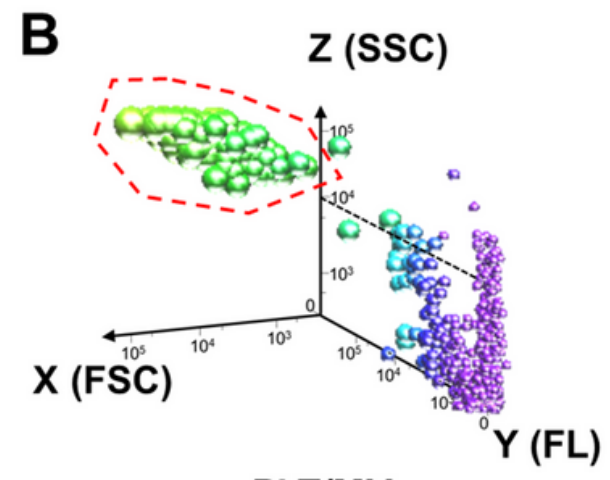

PLT/MV

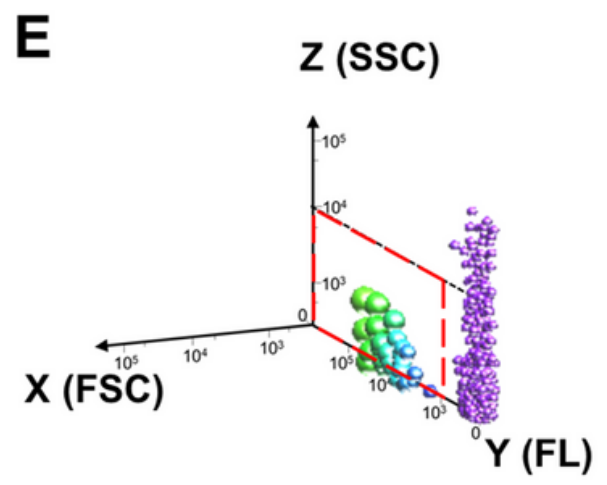

S2

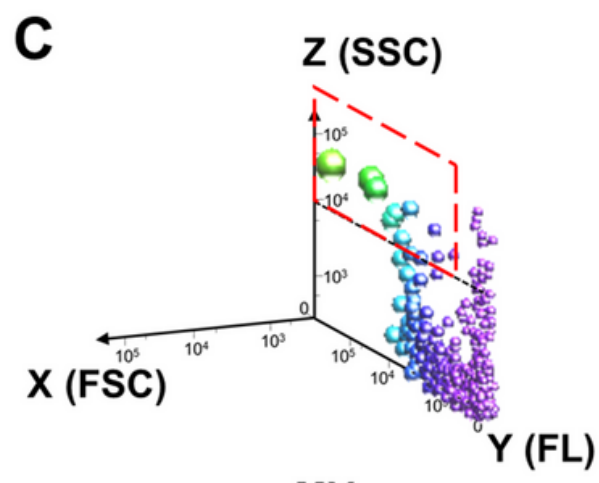

MV

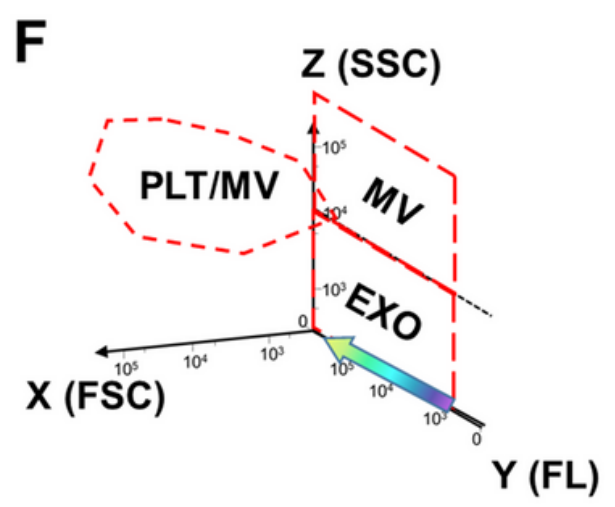

Gates 


\section{Figure 1}

Multiparametric analysis on platelet, microvesicle, and exosome-sized populations (A) Multiparametric analysis of Megamix-plus size calibration beads (100 nm to $900 \mathrm{~nm}$ in size). 3D axes include $x$-axis (forward scatter), $y$-axis (FL/A488), and z-axis (side scatter). Dotted line along y-axis (FL) indicates 200 $\mathrm{nm}$ size bead population at SSC of 104 intensity. (B) 3D plot of isolated platelets and platelet EVs expressing CD41a (FL/A405) gated on the subset of SSC > $200 \mathrm{~nm}$ with high FSC shown in dotted red region (PLT/MV). (C) 3D plot of MV populations revealed by CD41a (FL/A405) gated using SSC > $200 \mathrm{~nm}$ without FSC shown in dotted red box. (D) Single-spun plasma at $1000 \times \mathrm{g}$ (S1) showing residual platelet/MVs in dotted red region. (E) Double-spun plasma at 15,000 $\times \mathrm{g}$ (S2) showing loss of platelets and MVs with only residual exosome-sized populations with CD41a (FL/A405) expression gated by SSC < $200 \mathrm{~nm}$ in dotted red box. (F) Schematic of 3D gating strategies to define platelet/MVs with high FSC, microvesicle-sized EVs (MV) with low FSC, and exosome-sized EVs (EXO) with low SSC. 
A $\quad \mathrm{S} 1, \mathrm{RT}$
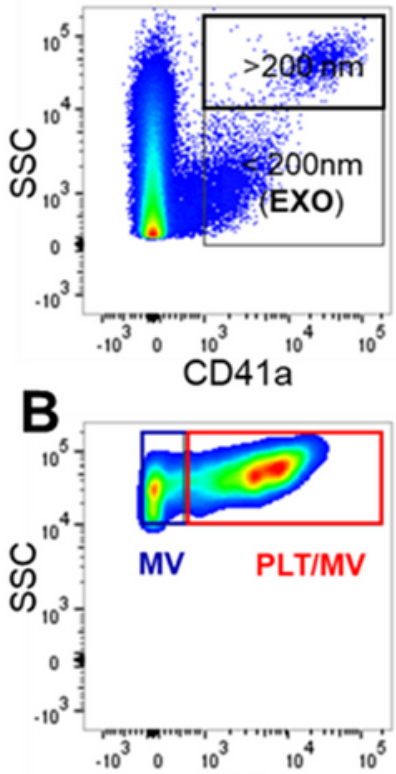

FSC
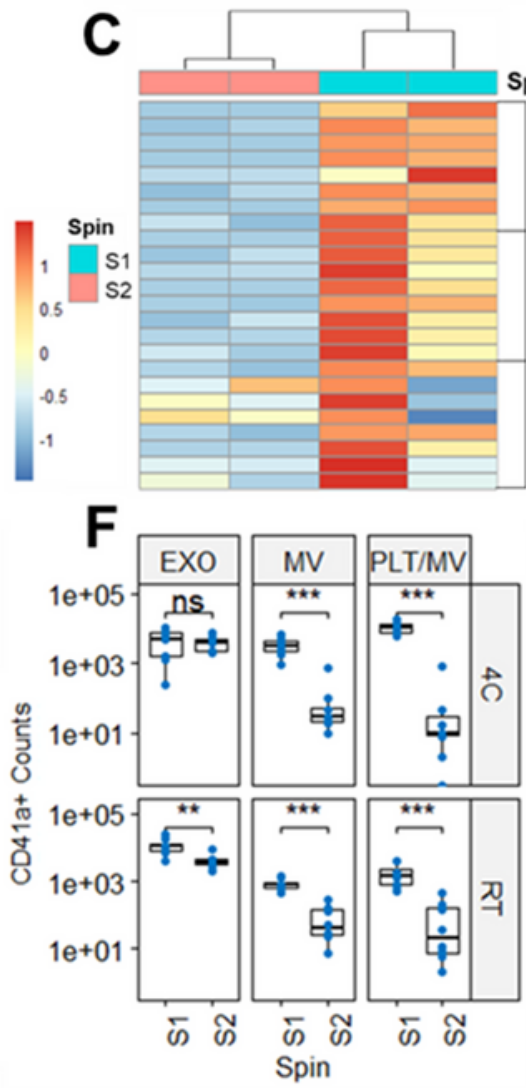

$\mathrm{S} 1,4 \mathrm{C}$
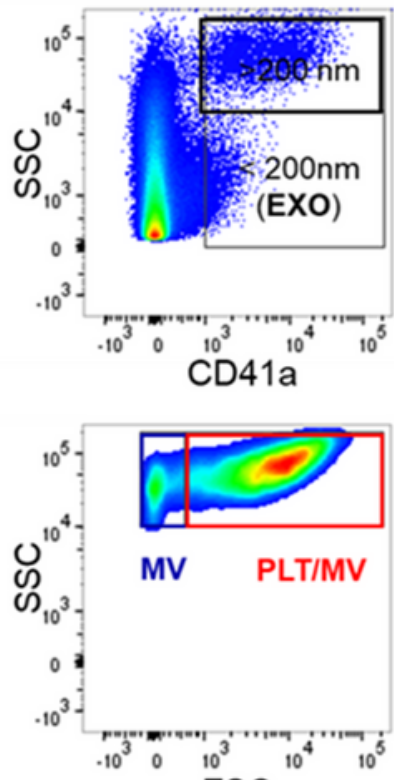

FSC
S2, RT
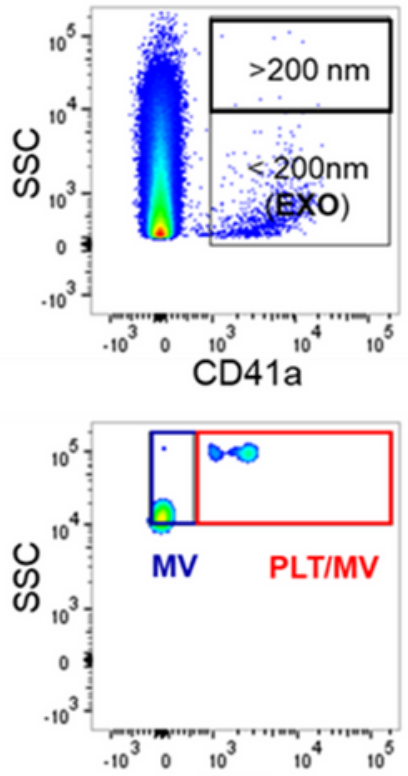

FSC

$\mathrm{S} 2,4 \mathrm{C}$
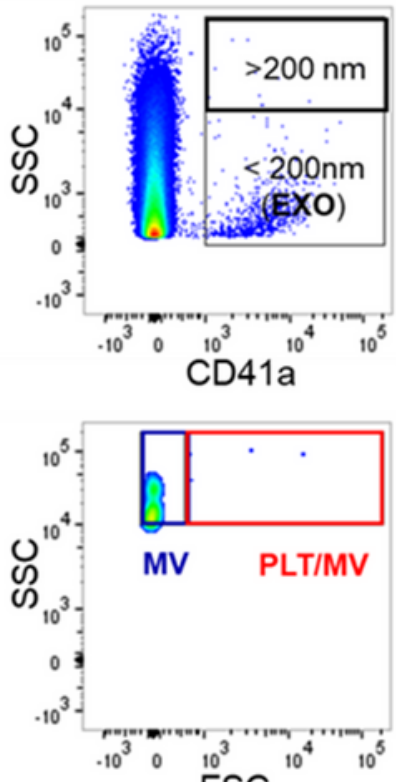

FSC
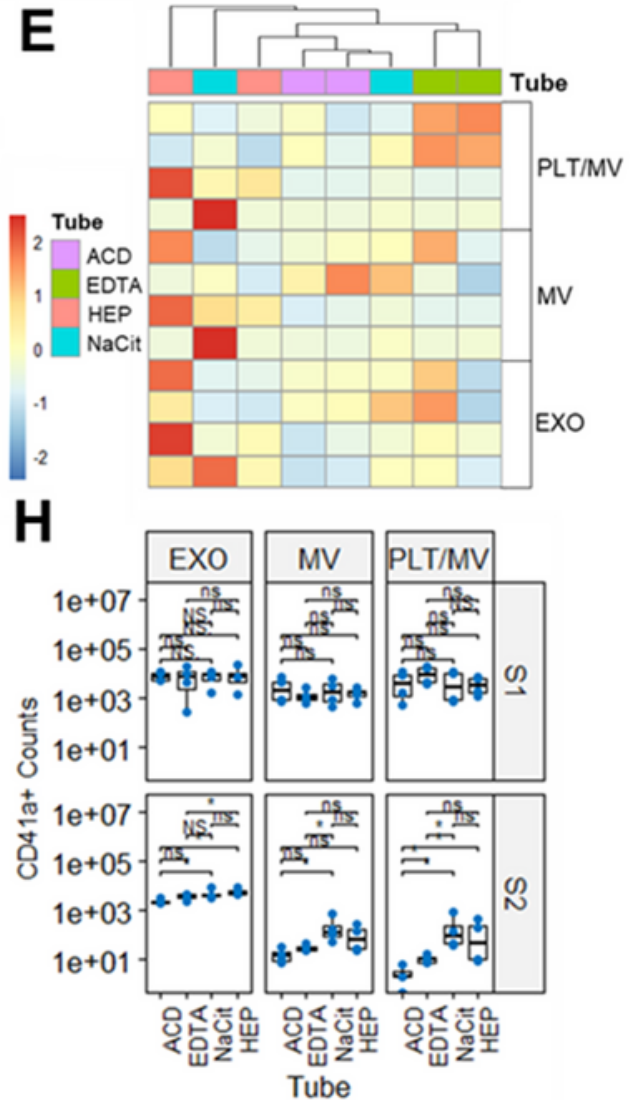

\section{Figure 2}

Identification of preanalytical influences on platelet and platelet-derived EVs (A) Representative flow cytometry dot plot of side scatter (SSC) versus CD41a+ fluorescent intensity (FL/A405) for: single spin at $1000 \times \mathrm{g}$ at either room temperature or at $4^{\circ} \mathrm{C}$ (S1 RT or S1 4C) and double spin at $15,000 \times \mathrm{g}$ at either room temperature or at $4^{\circ} \mathrm{C}$ (S2 RT or S2 4C). Gated areas enclosed SSC intensity above or below 104 (denoted as $200 \mathrm{~nm}$ size calibrated beads), wherein $<200 \mathrm{~nm}$ indicated gating for exosome-sized events 
(EXO). (B) Representative density plot of gated areas using SSC > $200 \mathrm{~nm}$ versus CD41a fluorescent intensity (FL/A405) back gated onto SSC versus FSC. Indicated gated regions denoted by platelets and MVs (PLT/MV) with high FSC in red and microvesicles (MV) with low FSC in blue. (C-E) Heatmaps of preanalytical influences (spin, temperature, or anticoagulant tubes) independently measured on PLT/MV and platelet derived EVs. (F-H) Box plots of average counts for PLT/MV, MV, and EXO with CD41a expressions were compared using pairwise Wilcoxon rank sum test (ns = not significant, $P>0.05 ; * P<0.05$, $\left.{ }^{\star} \mathrm{P}<0.01, * \star \star \star P<0.0001\right)$. Results are derived from two individuals' blood obtained in four different anticoagulant tubes and processed each at either room temperature or at $4{ }^{\circ} \mathrm{C}$.
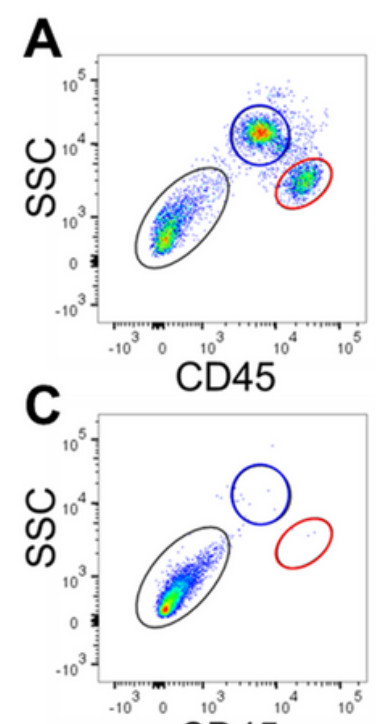

E

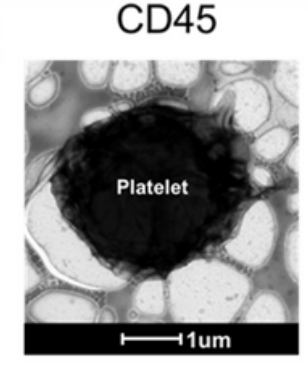

$\mathbf{F}$
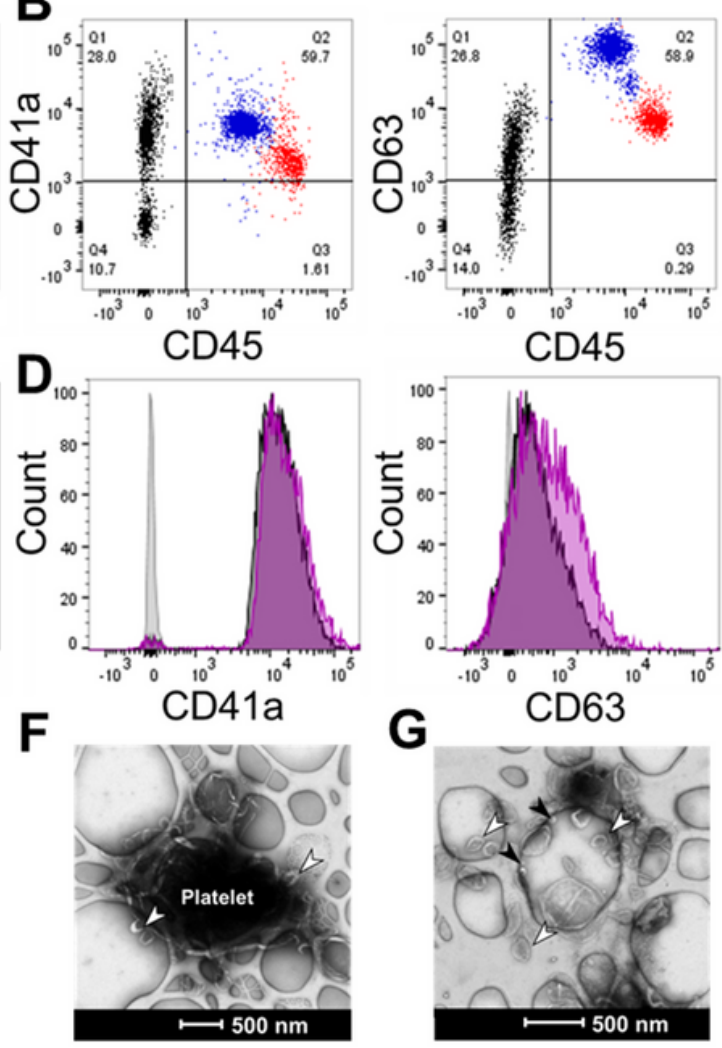

CD45

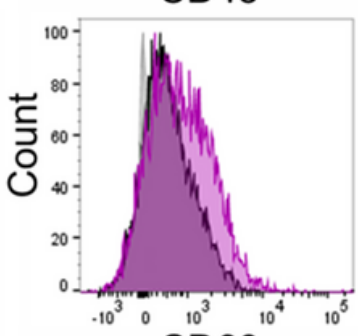

G

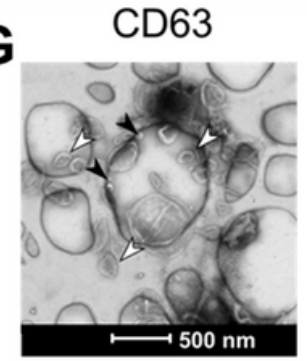

H

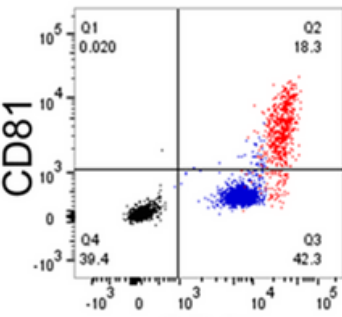

CD45
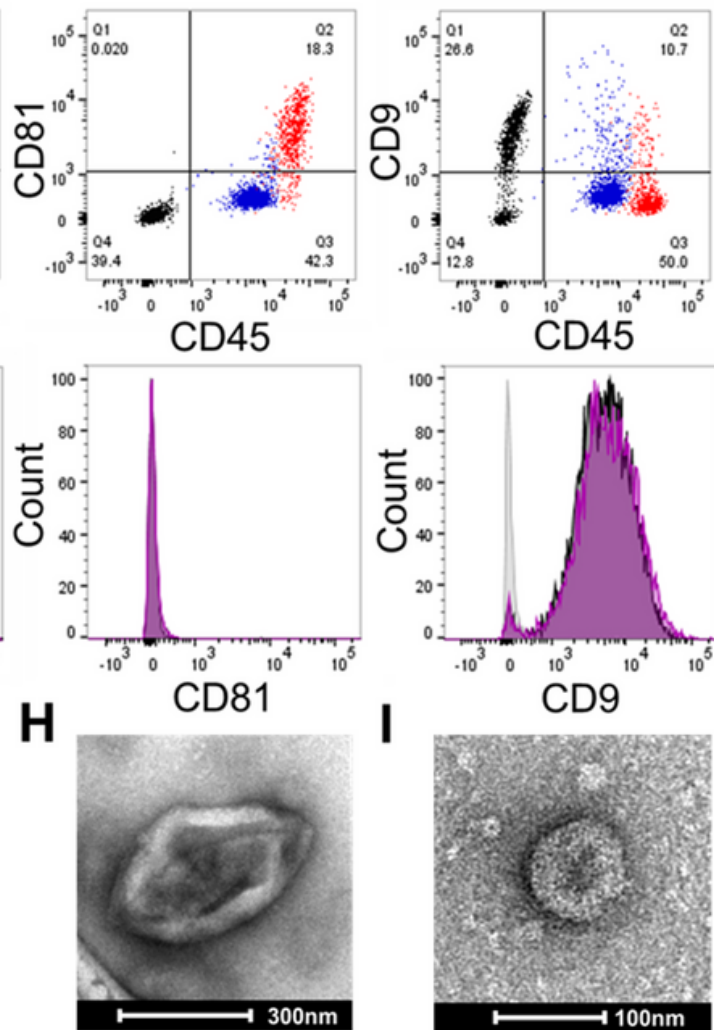

${ }^{10} \mathrm{CD} 45^{10^{\circ}}$

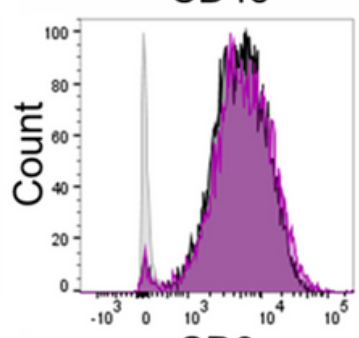

I
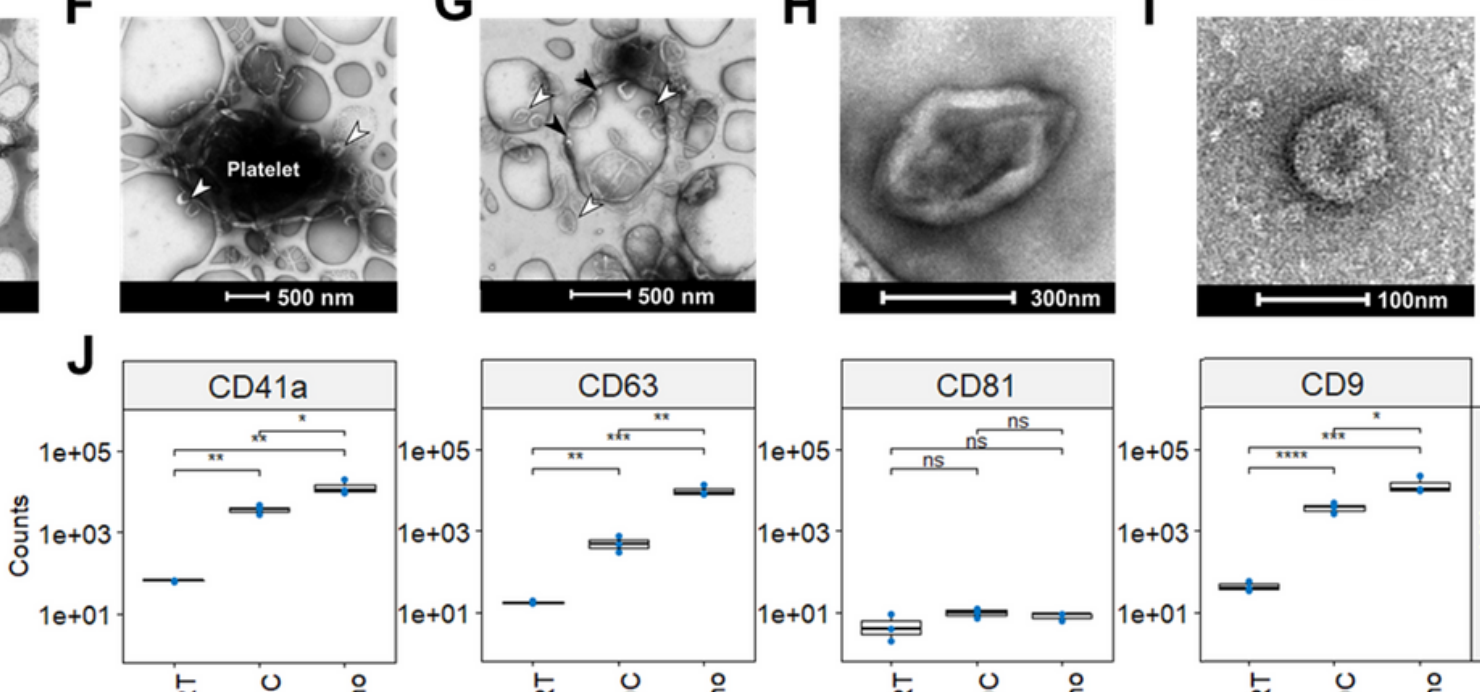

Size - MV

- EXO

Unstained PLT

Resting PLT

Activated PLT

Myeloid Cells

Lymphocytes
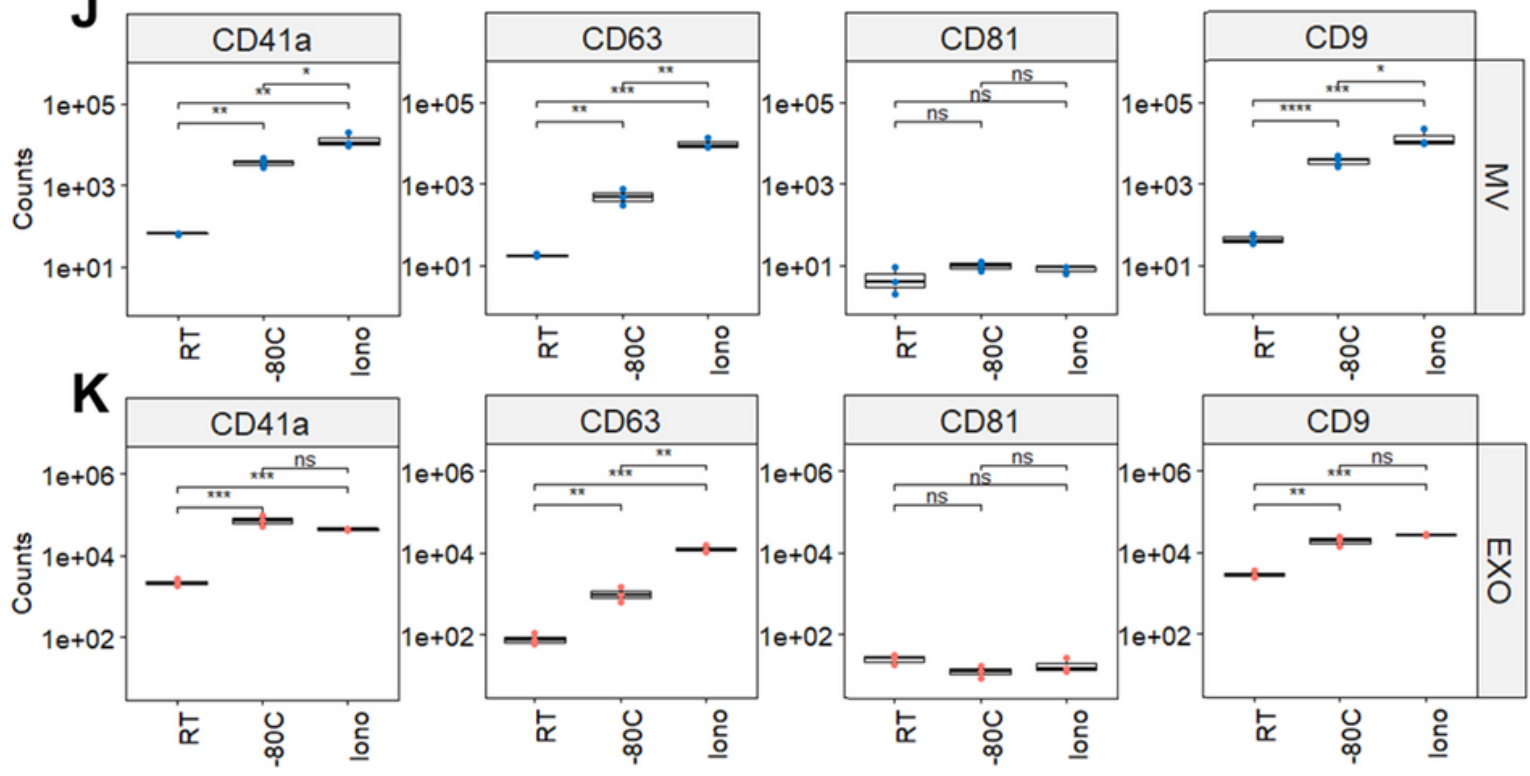

Figure 3 
Profiling isolated platelets and platelet-derived EVs by flow cytometry and TEM (A) Representative flow cytometry dot plot of side scatter (SSC) versus CD45 fluorescent intensity (FL/BV650) after red blood cell lysis, with gated areas indicating platelets (black), myeloid cells (blue), and lymphocytes (red). (B) Quadrant analysis on gated populations for individual markers (CD41a, CD63, CD81, or CD9) against CD45 (FL/BV650). (C) Dot plot of SSC versus CD45 (FL/BV650) on washed platelets with gated regions shown in a. (D) Flow cytometry median-mode histogram of the individual markers on resting platelets (black) and thrombin activated platelets (purple) with unstained controls overlaid in grey. TEM images of (E) resting platelet, $(F)$ activated and/or freeze fractured platelets banked at $-80^{\circ} \mathrm{C}$, and $(\mathrm{G})$ extracellular vesicles released from activated platelets showing $(\mathrm{H})$ microvesicles and $(\mathrm{I})$ exosome-sized particles. TEM scale bars as shown (E-I) with arrows indicating microvesicles (white) and exosomes (black). Box plots of $(J)$ platelet-derived MV counts derived from three technical replicates and $(K)$ EXO counts derived from three technical replicates for individual markers associated with platelet activations: $-80^{\circ} \mathrm{C}$ and ionomycin in the presence of $\mathrm{Ca} 2+$ (Iono) versus RT. Counts were compared by pairwise Wilcoxon rank sum test ( $\mathrm{ns}=$ not significant, $\mathrm{P}>0.05 ; * \mathrm{P}<0.05,{ }^{*} \mathrm{P}<0.01, * \star * * \mathrm{P}<0.0001$ ).
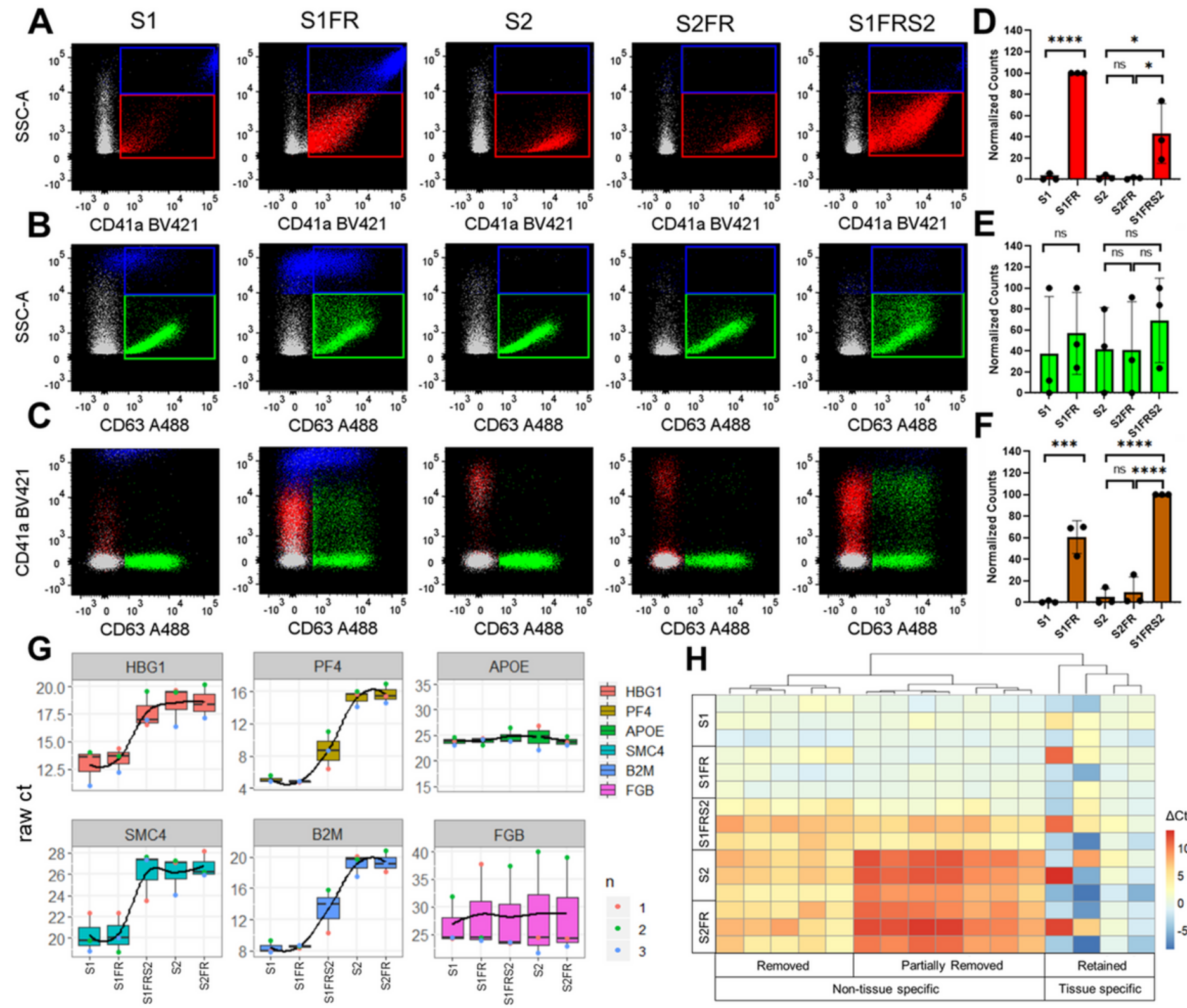

$\mathrm{H}$
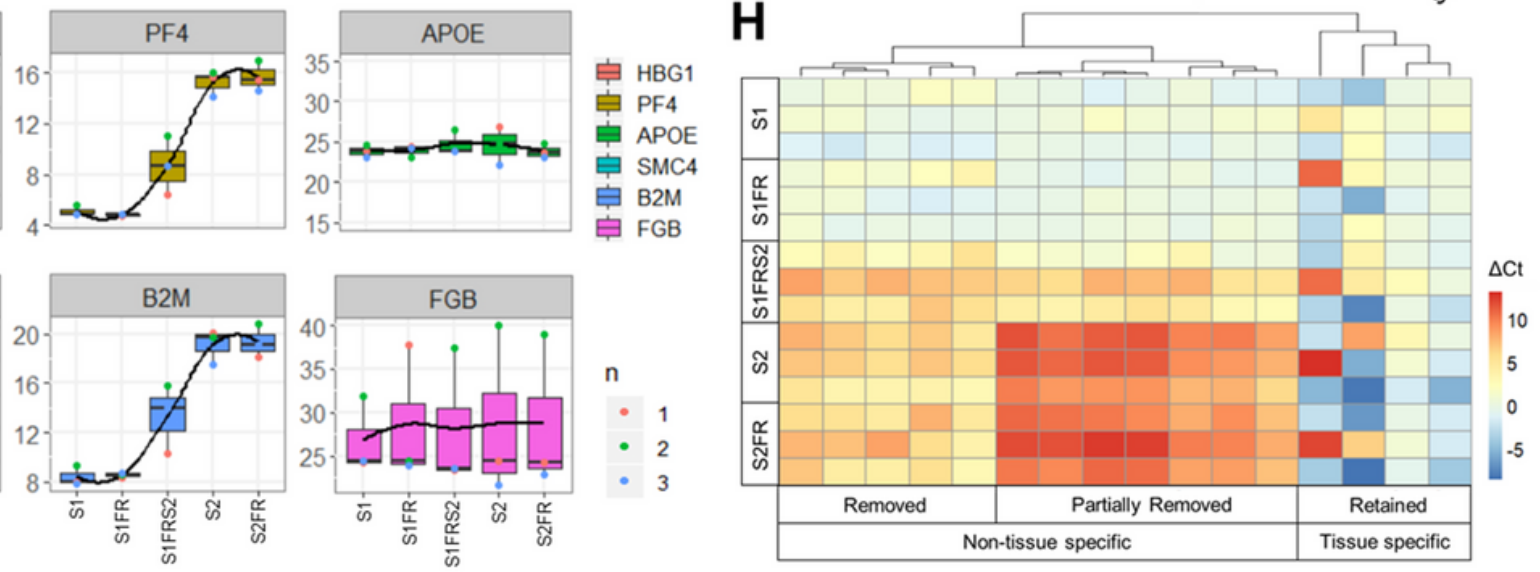


\section{Figure 4}

Effect of freeze thaw on EVs and cf-mRNA using flow cytometry and RT-qPCR (A-C) Representative scatter plots of EV phenotypes from S1, S2, respective subsequent freeze thaw processes (S1FR, S2FR), and secondary spin following S1FR (S1FRS2) for CD41a, CD63, and CD41a/CD63 costaining populations. Gated regions include PLT/MV and MV populations $>200 \mathrm{~nm}$ (blue), along with exosomesized subpopulations < 200nm with CD41a (red), CD63 (green), and CD41a/CD63 costaining (red/green). (D-F) Bar graph of normalized exosome counts measured in plasma samples from three healthy volunteers for each freeze thaw processing condition shown with corresponding marker colors were compared using Tukey's multiple comparisons (ns = not significant, $P>0.05 ; * P<0.05, * * * P<0.001$, $\star \star \star \star * P<0.0001)$. (G) Box plot of the median expression levels (in $C T s$ ) for representative non-tissue specific genes (HBG1, SMC4, PF4, and B2M) and tissue-specific genes (APOE, FGB) through freeze thaw processing conditions from three healthy volunteers using RT-qPCR. $(\mathrm{H})$ Hierarchical clustering analysis of relative amount (in $\Delta \mathrm{CT}$ ) representing non-tissue and tissue-specific genes. $\Delta \mathrm{CT}$ difference between S1 and individual post-thaw processing conditions from three healthy volunteers indicated from lowest (blue) to highest (red).

\section{Supplementary Files}

This is a list of supplementary files associated with this preprint. Click to download.

- SupplementaryFigures2020.12.19.docx 